\title{
Modular transmission line probes for microfluidic nuclear magnetic resonance spectroscopy and imaging
}

\author{
Manvendra Sharma ${ }^{\mathrm{a}}$, Marcel $\mathrm{Utz}^{\mathrm{a}}$ \\ ${ }^{a}$ School of Chemistry, University of Southampton, Southampton SO17 1BJ, United Kingdom
}

\begin{abstract}
Microfluidic NMR spectroscopy can probe chemical and bio-chemical processes non-invasively in a tightly controlled environment. We present a dual-channel modular probe assembly for high efficiency microfluidic NMR spectroscopy and imaging. It is compatible with a wide range of microfluidic devices, without constraining the fluidic design. It collects NMR signals from a designated sample volume on the device with high sensitivity and resolution. Modular design allows adapting the detector geometry to different experimental conditions with minimal cost, by using the same probe base. The complete probe can be built from easily available parts. The probe body mainly consists of prefabricated aluminium profiles, while the probe circuit and detector are made from printed circuit boards. We demonstrate a double resonance HX probe with a limit of detection of $1.4 \mathrm{nmol} \mathrm{s}{ }^{-1 / 2}$ for protons at $600 \mathrm{MHz}$, resolution of $3.35 \mathrm{~Hz}$, and excellent $\mathrm{B}_{1}$ homogeneity. We have successfully acquired ${ }^{1} \mathrm{H}_{-}{ }^{13} \mathrm{C}$ and ${ }^{1} \mathrm{H}_{-}{ }^{15} \mathrm{~N}$ heteronuclear correlation spectra (HSQC), including a ${ }^{1} \mathrm{H}-{ }^{15} \mathrm{~N}$ HSQC spectrum of $1 \mathrm{mM}{ }^{15} \mathrm{~N}$ labeled ubiquitin in $2.5 \mu \mathrm{l}$ of sample volume.
\end{abstract}

\section{Introduction}

NMR (Nuclear Magnetic Resonance) is a well established technique used in chemical analysis [1-3], molecular structure determination [4], metabolomics [5, 6], reaction monitoring [7] etc. Microfluidic lab-on-a-chip (LOC) devices are increasingly finding applications in chemistry and the life sciences $[8,9]$. They provide a convenient way to integrate complex chemical and biochemical processes. In particular, microfluidic technology allows detailed control over growth conditions in the culture of cells, cell aggregates, and small organisms [10-12]. Lab-on-a-chip devices can also enable high experimental throughput by parallelisation. In spite of its analytical power, NMR has so far only rarely been used in microfluidic systems. In general, information is extracted from microfluidic devices only at the end point of the experiment. Typically, this is done using fluorescent probes, which bind to one or a few analytes of interest. This approach can reach very high sensitivity, with limits of detection down to femtomolar concentrations. However, it is usually destructive, since the sample is affected by the addition of the marker, and can only be done once, at the end of the experiment. Following kinetic processes therefore requires using multiple samples. By contrast, NMR spectroscopy is non-invasive, generic (virtually all organic molecules give an NMR signal), and highly specific (no two metabolites share the same NMR spectrum), and can therefore provide a useful complementary readout capability for microfluidic devices. For example, the metabolic activity of cell cultures can be followed non-invasively by NMR throughout the course of the experiment [13], while effects on cell differentiation can still be analysed by a (destructive) transcriptomic analysis at the end. Unfortunately, the sensitivity of NMR is much lower than that of optical techniques. The small volumes involved in microfluidic systems (typically in the range of $1 \mu \mathrm{l}$ ) compound this problem. As is well known, the mass limit of detection of inductive NMR detectors improves as they are scaled down [14]. On this basis, a number of different micro-NMR detector designs have been proposed and characterised in the literature $[15,16]$. In most cases, these are based on micro-solenoid [17, 18], planar [19-21], or stripline detectors [22]. Significant research has gone into developing micro-NMR detectors for flow probes [2329], where the sample container takes the form of a fixed capillary into which the sample must be injected. Only a few designs that allow insertion of a removable microfluidic device into the detector have been described [30-33].

In this paper we present a novel modular probe designed for generic microfluidic NMR experiments. The design is based on a transmission line detector $[22,32]$ using inexpensive printed circuit board (PCB) technology. A modular design ensures that detectors of different geometry and size can be easily exchanged. The NMR detector (including the tuning and matching circuit) is connected to the probe skeleton through detachable connectors. Modularity of the probe not only allows fast optimisation of the detector but also facilitates the use of dedicated detectors for different microfluidic devices on a single probe base. Once the probe base is in place, additional detectors can be manufactured within 2-3 days for less than $\sim 200 €$. The probe is optimised for proton detected double resonance, with the second channel tunable to different nuclei such as ${ }^{13} \mathrm{C},{ }^{15} \mathrm{~N}$, or ${ }^{31} \mathrm{P}$. The probe skeleton is made almost 


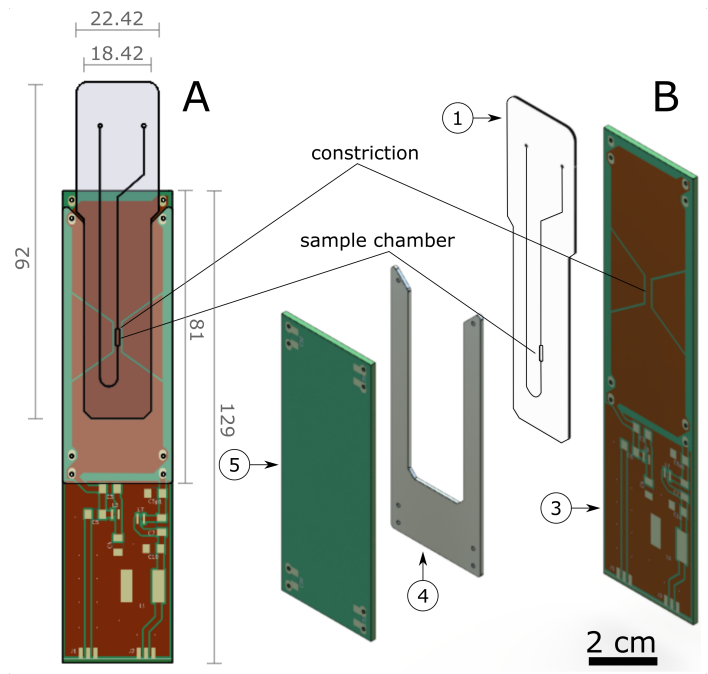

Figure 1: Drawings of the detector assembly and the microfluidic device (1). A: front view (dimensions in $\mathrm{mm}$ ); B: exploded view. Spacer (4) ensures the alignment of the sample chamber with the constrictions on the $\mathrm{PCB}$ planes. In $\mathrm{A}, \mathrm{PCB}$ plane 5 is hidden to show the orientation of 1 with respect to $\mathrm{PCB}$ plane 3 . Thickness of each of the PCB planes is $1.52 \mathrm{~mm}$ and the copper layers on the PCBs is $35 \mu \mathrm{m}$. Both the microfluidic device and the spacer are made from PMMA and have thickness of $0.9 \mathrm{~mm}$ and $1 \mathrm{~mm}$ respectively.

entirely from easily obtainable raw materials such as aluminium profiles of standard size, with only a small number of custom-machined parts holding the structure together. Detailed construction drawings and a complete set of CAD files is available for download at https://github.com/ marcel-utz/modular-microfluidic-probe. The planar geometry of the detector accommodates exchangeable microfluidic devices which can be designed freely for specific experimental requirements as long as the overall shape of the device is maintained. Fig. 1 shows the microfluidic device and the detector with their relative positioning.

In the remainder of this paper, we discuss the probe design in more detail, and characterise its performance in terms of sensitivity and resolution. We also demonstrate its application in microfluidic cell culture, protein NMR spectroscopy, and micro-imaging.

\section{Probe Design}

The probe assembly design is based on readily and easily available materials and parts with minimal need for customised fabrication. All mechanical and electronic components are readily available from commercial sources, with the exception of a small number of precision-machined parts which are used to hold the assembly together. These can be ordered from commercial prototyping manufacturers or machined in-house if mechanical workshop facilities are available.

The probe is composed of three main assemblies: A) probe base; B) scaffold assembly; C) detector assembly (Fig. 2). The base (blue in Fig. 2) and the scaffold assembly (green in Fig. 2) together make the skeleton of the

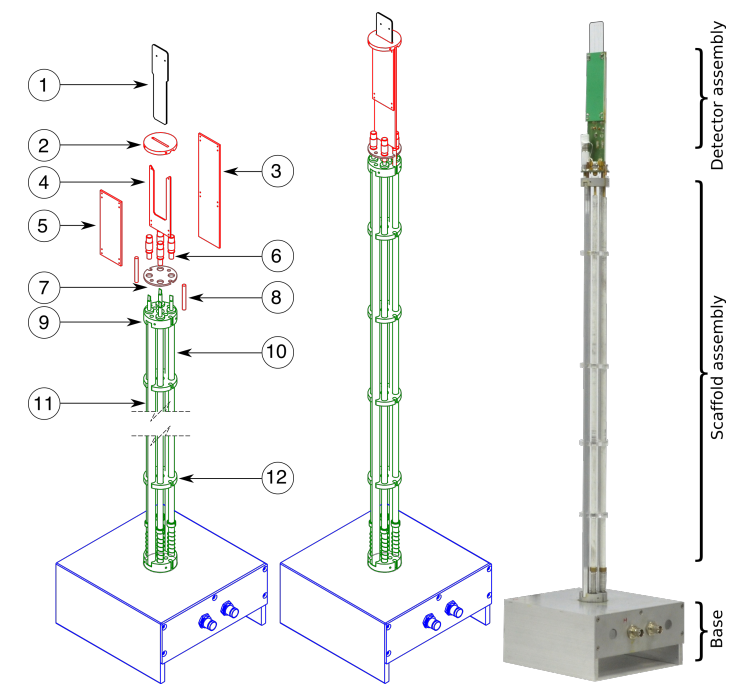

Figure 2: Exploded view of the probe design (left) and assembled probe (center). Probe base (blue) and scaffold assembly (green) with TMPCB (brown, 7) provides a generic mounting point for the NMR detector assembly (red). A microfluidic device (1) can be inserted from the top of the probe. The device is held in place by a spacer (4) between the detector planes ( 3 and 5 ) ensuring alignment of the sample chamber with the sensitive area on the PCB planes. The modular NMR detector is made up of parts 3,4 and, 5 and is connected to the TMPCB (7) through standard coaxial (SMP) connectors. The scaffold assembly is made up of aluminium tubes (11), scaffold support disks (9) and, auxiliary support disks (12).

probe. Both the base and scaffold assembly are made from standard aluminium profiles. The base contains the BNC connectors for RF (Radio Frequency) input. These BNC connectors are connected to semi-rigid coaxial cables for $\mathrm{RF}$ transport in the probe.

The scaffold assembly is made up of two hollow aluminium tubes (11) held together with precision machined parts at both ends (scaffold support disks (9)). The semirigid cables (RG 402, $50 \Omega$ ) are threaded through these hollow aluminium tubes to reach the detector assembly. Auxiliary support disks (12) provide additional rigidity for the scaffold assembly. In our case, these were laser cut from $5 \mathrm{~mm}$ thick poly(methyl methacrylate) (PMMA) sheets, but they may also be made by conventional machining. A tuning and matching PCB (TMPCB, 7) is held in place on the top of the probe skeleton by the detector support rods (8), with the semi-rigid coaxial cables providing additional support. The TMPCB is permanently fixed to the probe base, and serves as attachment point for the exchangeable detector assembly. It also provides trimmer capacitors (6) for secondary tuning and matching of the detector. The detector assembly is connected to the TMPCB through a pair of low-loss SMP connectors (14 in Fig. 3). The SMP connector receptacle male pin $\left(J_{3}\right.$ and $J_{4}$ in Fig. 5) is soldered on the TMPCB and the SMP connector jack male pin is soldered on the PCB plane 3 $\left(J_{1}\right.$ and $J_{2}$ in Fig. 5$)$. The TMPCB and 3 are connected by a coaxial connector SMP plug adapter. While each detector assembly is roughly tuned and matched with fixed 
circuit elements soldered onto it, the adjustable capacitors on the TMPCB are used for fine tuning and matching at the magnet through tuning rods (10) which reach all the way to the probe base.

The base, the scaffold assembly and the tuning and matching $\mathrm{PCB}$ all have a free circular axial bore providing access from the bottom of the probe, which can be used for temperature and environmental control and/or fluidic connections to the sample as needed. The detector assembly is made up of two PCBs (3 and 5) and a spacer (4) between them. The spacer is made from a $1 \mathrm{~mm}$ thick PMMA sheet. The detector used here is based on a planar transmission line geometry as described elsewhere [22, 32]; other structures such as spiral coils and Helmholtz pairs are also possible, and can easily be realised on a PCB. The transmission line detector consists of a pair of copper planes, each with a constriction at the centre. This geometry gives rise to an electromagnetic eigenmode with a strong anti-node of the magnetic field between the two planes at the site of the constriction. This concentrates the rf field and the detection sensitivity onto the sample area. The shape and size of the copper structures have been chosen such as to produce an eigenfrequency of this mode around $630 \mathrm{MHz}$. The PCBs have been designed with solder points at the back side for chip capacitors; these can be used to tune the resonance down to the desired proton Larmor frequency (500 or $600 \mathrm{MHz}$ in the present case). Both the PCBs (3 and 5) also carry circuitry for channel separation and primary matching of the detector. A copper or brass auxiliary disk (2) holds the PCBs together at the top, and forms the top end of the probe assembly. The probe sheath (not shown in Fig. 2) is fixed to the auxiliary disk (2) and the scaffold assembly with screws. An exchangeable microfluidic device (1) can be inserted from the top of the detector. Microfluidic devices can be used as passive sample holders, filled before putting them into the probe, or the device can be actively perfused and operated in-situ. The device inlet and outlet are sealed by an optical adhesive film (MicroAmp, AB Applied Biosystems) when using the device as passive sample holder. The microfluidic device is held in place by the spacer between the two PCB planes coinciding the sample chamber with the constriction on the detector. The 45-degree edges of the device outline help with precise and reliable alignment. Since the detector assembly is attached to the probe base through SMP connectors, it can easily be exchanged. Using standard PCB processes to manufacture the detector provides great flexibility to optimise detector performance for different applications, including different microfluidic sample sizes, and different combinations of target nuclei.

Fig. 3 shows two different versions of the probe with and without the RF insert (13) for the X channel. In (A) the $\mathrm{X}$ channel can be tuned to different nuclei by exchanging (13). However, in (B) the circuitry of the RF insert (13) is integrated on the PCB plane (5), making the design more compact. Probe (A) is compatible with a Varian 600 Premium shielded 14.1 T magnet, with an outer diameter

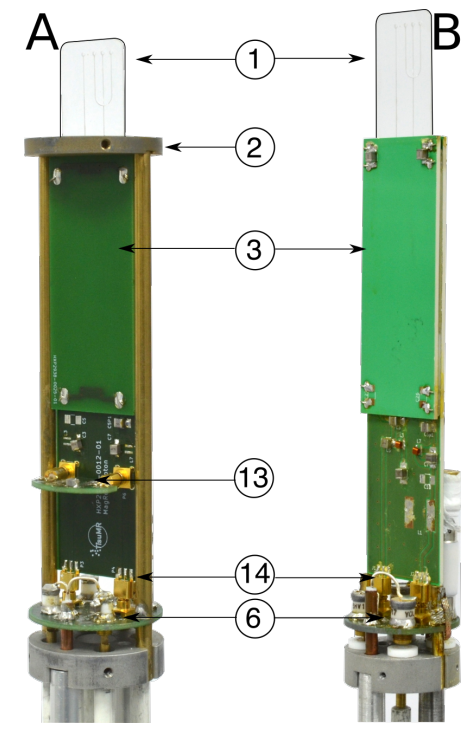

Figure 3: Dual channel probes with (A) and without (B) modular $\mathrm{RF}$ insert (13) for $\mathrm{X}$ channel. O.D. of $\mathrm{A}$ is $44.45 \mathrm{~mm}$ and $\mathrm{B}$ is 38 $\mathrm{mm}$. Circuit elements of $\mathrm{X}$ channel are integrated on the PCB in B making it compact. Detachable SMP connectors (14) are used to connect the RF insert (13) and the detector assembly.

(O.D.) of $44.45 \mathrm{~mm}$ including the sheath. Probe (B), with an O.D. of $38 \mathrm{~mm}$ including the sheath, is designed to fit inside a Bruker narrow bore shim stack. Probe (B) also fits inside a Bruker micro-imaging gradient unit. Naturally, B can also be used with wide bore magnets with appropriate spacers to keep the probe centered.

The microfluidic devices were made from inexpensive PMMA sheets by laser cutting and thermal bonding, as described in detail elsewhere [34]. PMMA was chosen for ease of fabrication, but also due to the close match in magnetic susceptibility with that of water (Table 1). Briefly, the devices consist of a $0.5 \mathrm{~mm}$ thick middle layer between a top and bottom layer of $0.2 \mathrm{~mm}$ thickness each. The sample chamber and fluidic network are cut into the middle layer. The fluidic network on the device can be designed freely as long as the overall shape of the device is preserved, and the location of the sample chamber is maintained. The volume of the sample chamber is $2.5 \mu \mathrm{l}$ which can be increased or decreased according to the experimental requirements. Fig. 4 shows various microfluidic devices.

Table 1: Magnetic susceptibility values of some relevant materials. [35, 36]

\begin{tabular}{||l|l||}
\hline Material & $\chi / \mathrm{ppm}$ \\
\hline PMMA & -9.054 \\
Copper & -9.63 \\
FR4 & -3.743 \\
Aluminium & 20.7 \\
Water & -9.02 \\
\hline
\end{tabular}

The double resonance circuit is based on the design shown in Fig. 5.The transmission line detector is tuned 


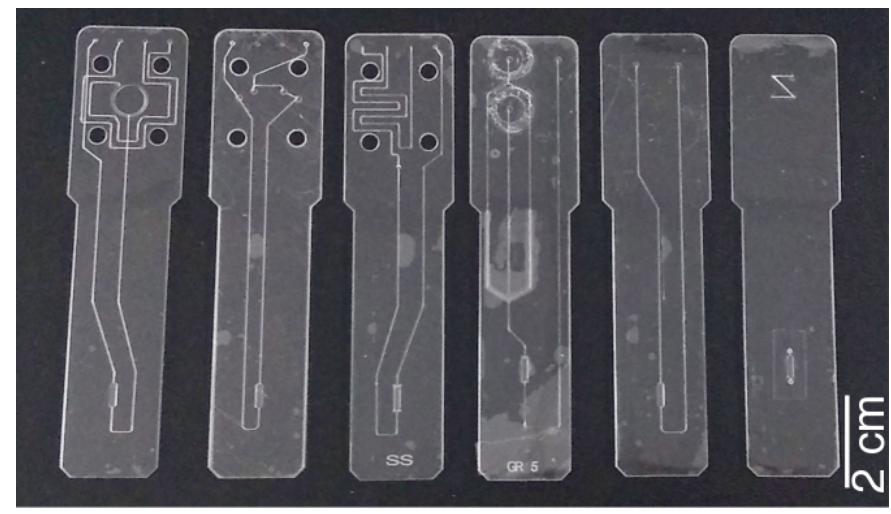

Figure 4: Microfluidic devices for different applications compatible with the current setup. From left: Devices for perfusion of tissue slice on a chip, peristaltic pumping to induce flow, hydrogenation on a chip, droplet generator, simple design to fill the sample chamber and, to grow cells on the device.

to the desired proton Larmor frequency by chip capacitors $\left(C_{20}\right)$ located at its four corners, as well as a non-magnetic trimmer capacitor $\left(C_{6}\right)$. Two primary matching capacitors $\left(C_{5}\right.$ and $\left.C_{5}^{\prime}\right)$ bring the impedance into the vicinity of $50 \Omega$. A short microstrip with a specific impedance of $50 \Omega$ on the PCB then provides a connection to the secondary tuning and matching elements on the TMPCB $\left(C_{1}\right.$ and $\left.C_{2}\right)$. The lower frequency channel is connected to the detector through a high-frequency band reject $\left(C_{7}, L_{7}\right)$. Tuning is achieved by $L_{1}$ and $C_{4}$ in combination with the complex admittance of the detector at the $\mathrm{X}$ channel frequency. To maximise efficiency, $L_{1}$ should have as high a $Q$-factor as possible. We have used commercially available flat copper coils of $5 \mathrm{~mm}$ diameter and 4.5 turns in the case of ${ }^{15} \mathrm{~N}$ at $60 \mathrm{MHz}$. Tuning to ${ }^{13} \mathrm{C}$, either at 125 or at $150 \mathrm{MHz}$ could be achieved without $L_{1}$. Details of all the circuit elements are given in table 2 .

Table 2: Details of the circuit elements to tune the probe at 600 $\mathrm{MHz}$ and $60 \mathrm{MHz}$.

\begin{tabular}{||l|l|l||}
\hline Circuit element & Value & $\begin{array}{l}\text { Manufacturer } \\
\text { Part Number }\end{array}$ \\
\hline$C_{1}, C_{2}$ and, $C_{8}$ & $1-23 \mathrm{pF}$ & NMAM25HV \\
$C_{9}$ & $3-23 \mathrm{pF}$ & NMNT23-6 \\
$C_{6}$ & $3-23 \mathrm{pF}$ & NMAJ25HV \\
$C_{20}$ & $2.4 \mathrm{pF}$ & S111TUE \\
$C_{3}, C_{7}$ & $4.3 \mathrm{pF}$ & S111TUE \\
$C_{4}$ & $82 \mathrm{pF}$ & S111TUE \\
$C_{5}, C_{5}^{\prime}$ & $2.4 \mathrm{pF}$ & S111TUE \\
$L_{1}$ & $111 \mathrm{nH}$ & $1010 \mathrm{VS}-111 \mathrm{ME}$ \\
$L_{3}, L_{7}$ & $16.6 \mathrm{nH}$ & 17 NGLB \\
$J_{1}, J_{2}$ & - & 0734153591 \\
$J_{3}, J_{4}$ & - & SMP-MSLD- \\
\multicolumn{2}{|l}{} & PCT-5 \\
\hline
\end{tabular}

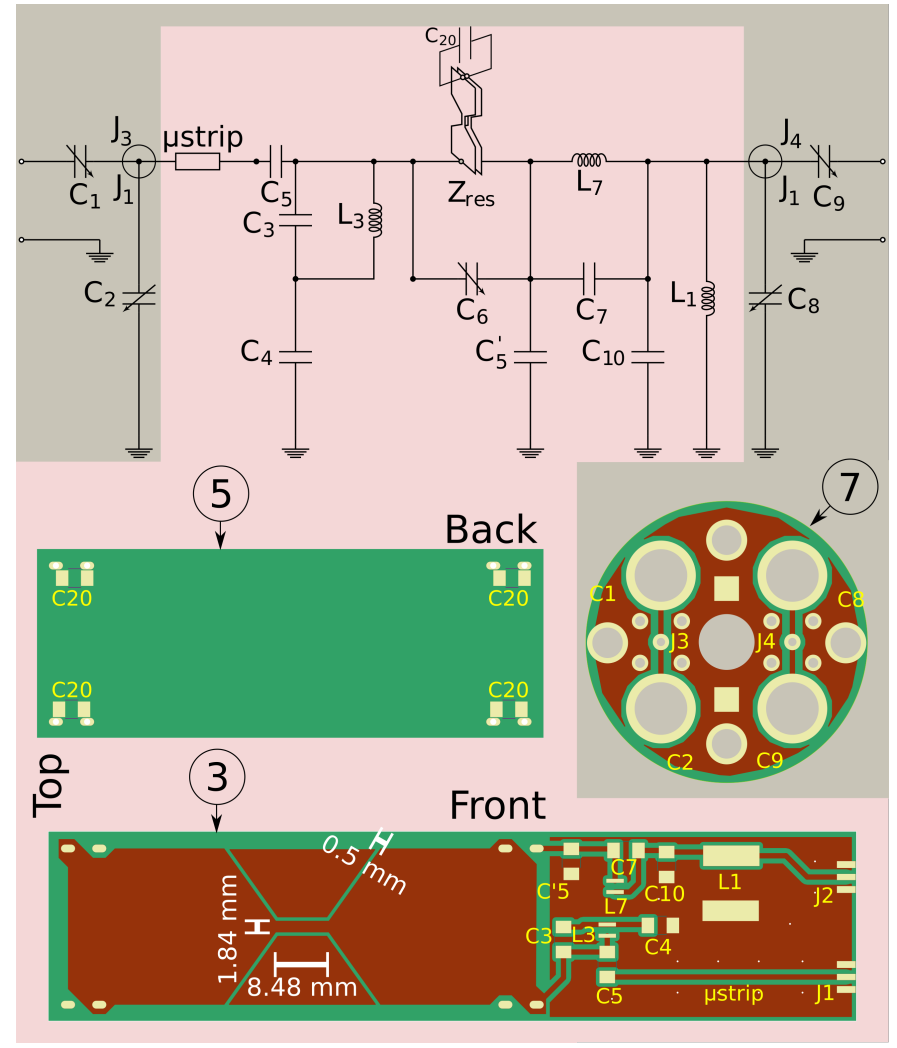

Figure 5: Circuit diagram of the double resonance probe. The background colour relates the different circuit elements on different PCBs. The back side of the PCB plane 5 and the front side of the PCB plane 3 is shown in horizontal orientation. The front side of the TMPCB 7 is shown in vertical orientation. Trimmer capacitor $C_{6}$ is placed on the back side of PCB plane 3. The copper layer is shown in brown and the solder pads to place the circuit elements are shown in off-white. An insulating solder mask layer over the copper layer prevents accidental short-circuiting. 


\section{Materials and Methods}

All the parts and the probe assembly were designed in Solidworks (Solidworks, Dassault Systèmes). Prefabricated profiles made from 6061-T3 aluminium (Aluminum Warehouse, UK) were used for the mechanical structure of the probe (base platform, scaffold tubes, sleeve). A few customised precision-machined parts (scaffold support disks, top disk) were obtained from ProtoLabs (Telford, UK) after providing CAD files. A small number parts were machined in-house (detector support rods, auxiliary support disks). All electronic components including semi-rigid transmission lines were purchased from DigiKey Ltd and Farnell element14, UK. Sodium acetate and ${ }^{15} \mathrm{~N}$ labeled urea were purchased from Sigma-Aldrich, UK and Dulbecco Modified Eagle's Medium (DMEM) supplemented was purchased from Life Technologies, USA. Cell cast PMMA sheets for microfluidic devices were bought from Weatherall Equipment (Wendover, UK). Microfluidic devices were designed on AutoCAD. PMMA sheets were cut to desired size and functionality (holes or channels) using a laser engraving system (L3040 from HPC Laser LTD, Elland, UK), and then bonded using the protocol described in [34].

The precise dimensions of the detector were optimised for a given sample volume using finite element calculations in COMSOL, as described in [32]. The RF response of the probe circuit was simulated using a symbolic network analysis code [37] in order to estimate appropriate component values. Final tuning was done by trial and error using an Agilent FieldFox $4 \mathrm{GHz}$ network analyser. PCB layouts were designed using KiCAD, and then sent to beta-layout GmbH (Aarbergen, Germany) and P.W. Circuits Ltd. (Wigston, UK) for manufacturing. Easily and inexpensively available FR4 PCB material (Fig. 3(A)) proved adequate for some purposes, but limited resolution to about $10 \mathrm{~Hz}$. A high-quality Teflon-based RF PCB material (RO3035, Rogers Corp., USA) was therefore used for optimal performance in both sensitivity and resolution (Fig. 3(B)). The probe skeleton was assembled from prefabricated aluminium tubes held together with the precision-machined scaffold disks using brass screws. Semi-rigid transmission lines were then threaded through the tubes, and the TMPCB plate was soldered on top. The detector planes (3 and 5) and the PMMA spacer between them were assembled using short bits of $0.5 \mathrm{~mm}$ diameter copper wire bought from RS components, UK, which were soldered to the appropriate pads in the four corners of the detector structure.

\subsection{Details of NMR and MRI measurements}

NMR spectra were obtained on a Varian VNMRS console attached to a Varian 600 premium shielded $14.1 \mathrm{~T}$ magnet. Field maps in the Fig. 7 were created by gradient echo (FLASH) pulse sequence with echo times $\left(t_{e}\right)$ of 6 and $16 \mathrm{~ms}$ and repetition time $\left(t_{r}\right)$ of $100 \mathrm{~ms}$ on a sample of $130 \mathrm{mM}$ sodium acetate dissolved in $\mathrm{H}_{2} \mathrm{O}$. The peak shown in the Fig. 8 is of $\mathrm{H}_{2} \mathrm{O}$ in an aqueous solution of $100 \mathrm{mM}{ }^{15} \mathrm{~N}$-urea. Fig. 9 shows proton spectrum of $2.5 \mu \mathrm{l}$ of $130 \mathrm{mM}$ sodium acetate dissolved in $\mathrm{H}_{2} \mathrm{O}$. The spectrum was recorded after pre-saturating the water signal at a nutation frequency of $100 \mathrm{~Hz}$. 32 transients were averaged with a repetition delay of $3 \mathrm{~s}$. The proton spectrum of DMEM (Dulbecco modified Eagle's medium) cell growth medium in Fig. 10 was acquired with 256 scans in 20 mins. The left panel of Fig. 11 shows a ${ }^{13} \mathrm{C}^{-1} \mathrm{H}$ heteronuclear single quantum correlation (HSQC) spectrum, acquired in 12 mins ( $32 t_{1}$ increments and 16 scans) from a $2.5 \mu \mathrm{l}$ sample of $100 \mathrm{mM}{ }^{13} \mathrm{C}$-glucose dissolved in water. The right panel shows a ${ }^{15} \mathrm{~N}-{ }^{1} \mathrm{H}-\mathrm{HSQC}$ spectrum, acquired in 400 mins $\left(64 t_{1}\right.$ increments and 128 scans) from $2.5 \mu \mathrm{l}$ sample of $1 \mathrm{mM}{ }^{15} \mathrm{~N}$-ubiquitin $(17 \mu \mathrm{g})$ dissolved in $10 \mathrm{mM}$ phosphate buffer at $\mathrm{pH} 7$.

For MRI experiments precision-cut murine liver slices (PCLS) were obtained by first extracting a tissue core of $8 \mathrm{~mm}$ diameter using a biopsy punch. PCLS of 250-300 $\mu \mathrm{m}$ thickness were cut from the core using a Krumdieck Tissue slicer, and then reduced to $3 \mathrm{~mm}$ diameter using a smaller punch. The slices were incubated in well-plates at $37^{\circ} \mathrm{C}$ and $80 \% \mathrm{O}_{2}, 5 \% \mathrm{CO}_{2}$ for 2 hours before imaging. Imaging experiments were performed on a Bruker AVANCE III spectrometer on a Bruker Active Shield II wide bore $11.7 \mathrm{~T}$ magnet equipped with a Bruker Micro2.5 gradient system. The image shown in Fig. 12(B) was acquired using a FLASH pulse sequence with $t_{R}=120 \mathrm{~ms}$ and $t_{E}=15.3 \mathrm{~ms}$. The slice thickness of $1 \mathrm{~mm}$ encompasses the entire thickness of the tissue. Fig. 12(C) shows a RARE image using the same parameters, with 8 echos acquired per scan, thus reducing the acquisition time to 8 minutes. A spin echo image is shown in Fig. 12(D), acquired over $25 \mathrm{~min}$. Finally, Fig. $12(\mathrm{E})$ shows a $B_{0}$ field map, which has been obtained by acquiring two separate FLASH images with echo times $t_{E}=5.3 \mathrm{~ms}$ and $15.3 \mathrm{~ms}$, respectively.

\section{Results and Discussion}

The probe is designed for double resonance, with protondetected experiments in mind. The circuit is optimised for efficiency and sensitivity at the high-frequency channel, while allowing RF pulses to be applied at the $\mathrm{X}$ frequency as well. As already explained, the $\mathrm{X}$ channel tuning frequency can be changed by simply exchanging either only a small RF-insert or the complete detector assembly. Fig. 6 shows the measured reflection and transmission scattering coefficients ( $S$-parameters) as a function of frequency for two combinations of proton $(600 \mathrm{MHz}, 500 \mathrm{MHz})$ and $\mathrm{X}$ channel frequencies $(60 \mathrm{MHz}$ and $125 \mathrm{MHz}$ ), corresponding to the Larmor frequencies of ${ }^{15} \mathrm{~N}$ and ${ }^{13} \mathrm{C}$ at magnetic fields of $14.1 \mathrm{~T}$ and $11.7 \mathrm{~T}$ respectively. The measurements were performed in the pairs of $600 \mathrm{MHz}$ and $60 \mathrm{MHz}$ and $500 \mathrm{MHz}$ and $125 \mathrm{MHz}$. The loaded $Q$ factors, determined from the $-3 \mathrm{~dB}$ points of the reflection curves, are 42,49 , 

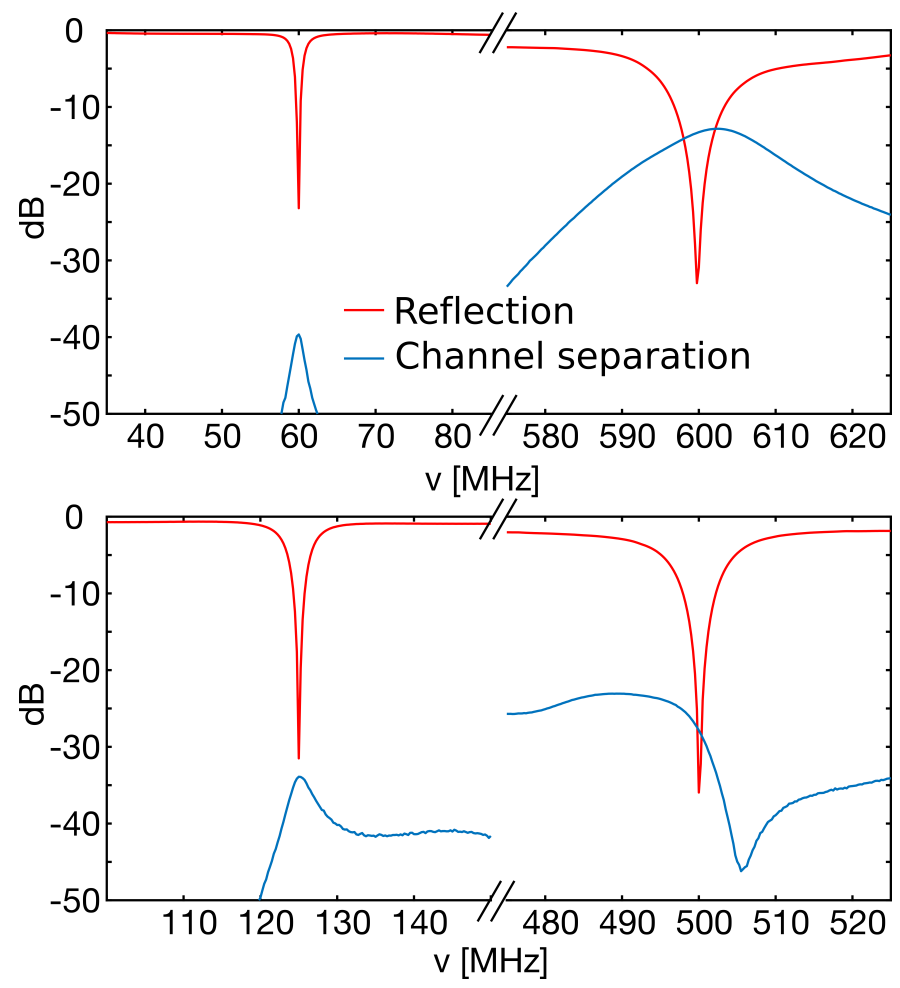

Figure 6: Experimental tuning and matching curves measured at $600 \mathrm{MHz}, 500 \mathrm{MHz}, 60 \mathrm{MHz}\left({ }^{15} \mathrm{~N}\right.$ frequency at $\left.14.1 \mathrm{~T}\right)$ and $125 \mathrm{MHz}$ $\left({ }^{13} \mathrm{C}\right.$ frequency at $\left.11.7 \mathrm{~T}\right)$. Probe can be tuned to 2 frequencies at a time, above results were measured in pairs of $600 \mathrm{MHz}$ with $60 \mathrm{MHz}$ and $500 \mathrm{MHz}$ with $125 \mathrm{MHz}$. Decoupling can be performed at all the frequencies as the channel separation is better than 15 decibels at all frequencies.

32, 36 at $600 \mathrm{MHz}, 500 \mathrm{MHz}, 125 \mathrm{MHz}$ and, $60 \mathrm{MHz}$ respectively. The channel separation is better than $-15 \mathrm{~dB}$ at all operating frequencies.

Initially, FR4 PCB material was used for the detector assembly because of its low cost and easy availability. Inhomogeneities in the $\mathrm{B}_{0}$ field due to the glass fibre rovings in FR4's composite backing can be seen as fringes in Fig. 7 (A). $B_{0}$ field maps of the sample chamber were constructed from 2 gradient echo (FLASH) images for both FR4 and RO3035 PCB materials with the same chip (Fig. 7). The brightness of each pixel is proportional to the sum of the magnitude of the two images, whereas the colour encode the phase difference between them. The full colour range from red to blue spans phase differences from $-\pi$ to $+\pi$. $\mathrm{B}_{0}$ field map of RO3035 PCB material clearly show better $\mathrm{B}_{0}$ field homogeneity compare to FR4. To avoid the inhomogeneities in FR4 and therefore to achieve better resolution, detectors were made from RO3035. While RO3035 provides better resolution, it is more expensive and takes longer to obtain compared to FR4. It therefore makes sense to first prototype new detector designs in FR4, and only use the more expensive RO3035 once the design has been validated.

The RF field at proton frequency shows excellent ho-
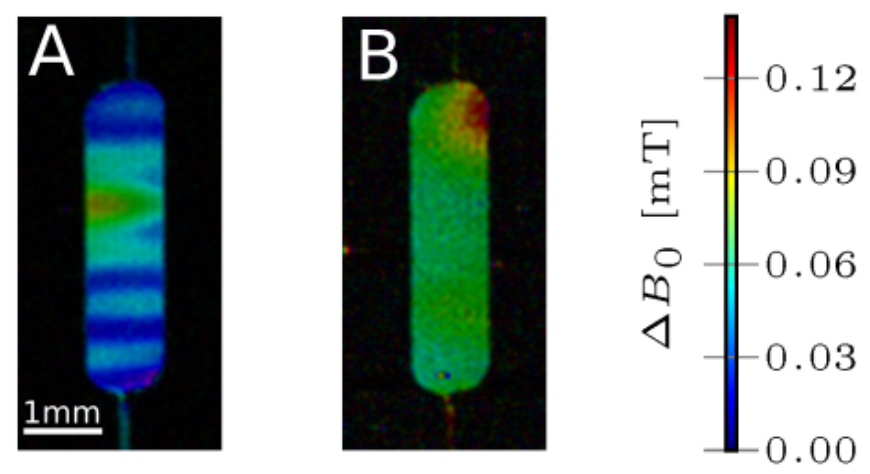

Figure 7: $\mathrm{B}_{0}$ field map of the sample chamber for FR4 (A) and Rogers RO3035 (B) PCB material acquired at 11.7 T. Both the field maps were constructed from 2 gradient echo images acquired on a chip filled with $130 \mathrm{mM}$ sodium acetate dissolved in $\mathrm{H}_{2} \mathrm{O}$ through same parameters and pulse sequence.

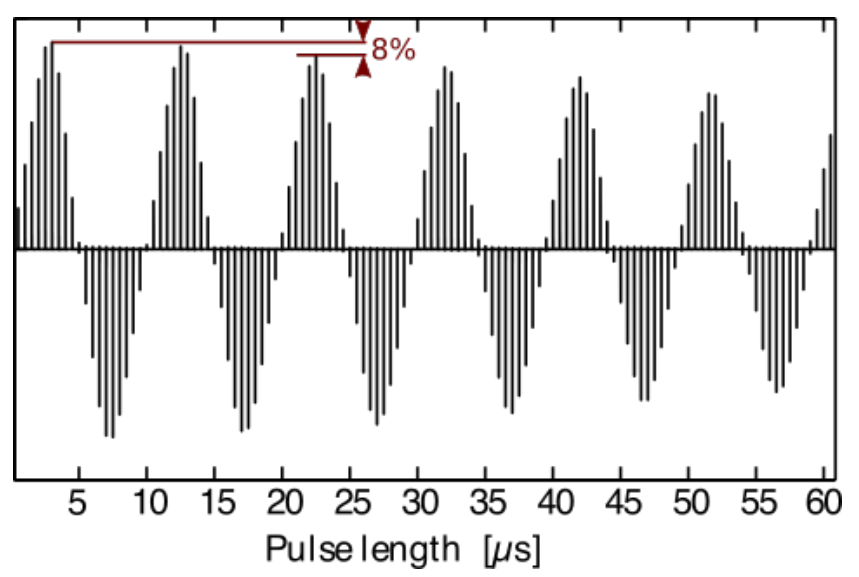

Figure 8: ${ }^{1} \mathrm{H}$ nutation acquired for water peak from a sample of $100 \mathrm{mM}{ }^{15} \mathrm{~N}$-urea dissolved in water measured at $600 \mathrm{MHz}$. The $810^{\circ} / 90^{\circ}$ ratio is $92 \%$ at $600 \mathrm{MHz}$. The $90^{\circ}$ pulse length is below $2.5 \mu \mathrm{sec}$ corresponding to $100 \mathrm{kHz}$ radio-frequency field for an input power of $100 \mathrm{~W}$. Similar $\mathrm{B}_{1}$ efficiency and homogeneity was observed at $500 \mathrm{MHz}$.

mogeneity, with a $810^{\circ} / 90^{\circ}$ ratio of more than $90 \%$ measured from ${ }^{1} \mathrm{H}$ nutation at $600 \mathrm{MHz}$ shown in Fig. 8. The probe generates $100 \mathrm{kHz}$ nutation frequency from $100 \mathrm{~W}$ input power both at $600 \mathrm{MHz}$ and $500 \mathrm{MHz}$, corresponding to RF field efficiency $\left(B_{1} / \sqrt{\text { power }}\right)$ of $10 \mathrm{kHz} / \sqrt{W}$. The detector geometry has been optimised for a sample volume of $5 \mu \mathrm{l}$. It compares reasonably well to the value of $22 \mathrm{kHz} / \sqrt{W}$ that has been reported by Finch et al. [32] for single-frequency detector of half this size at a Larmor frequency of $300 \mathrm{MHz}$. For the X channel, $100 \mathrm{~W}$ of input power produces nutation frequencies of $6 \mathrm{kHz}$ for ${ }^{15} \mathrm{~N}$ and $14 \mathrm{kHz}$ for ${ }^{13} \mathrm{C}$. No signs of arcing were obseved at this power level for pulses up to $1 \mathrm{~ms}$ duration.

Fig. 9 shows the proton spectrum of sodium acetate. The inset shows an expanded view of the base of the acetate peak. Carbon satellites can be seen well above the noise level. The orange curve corresponds to a Lorentzian 


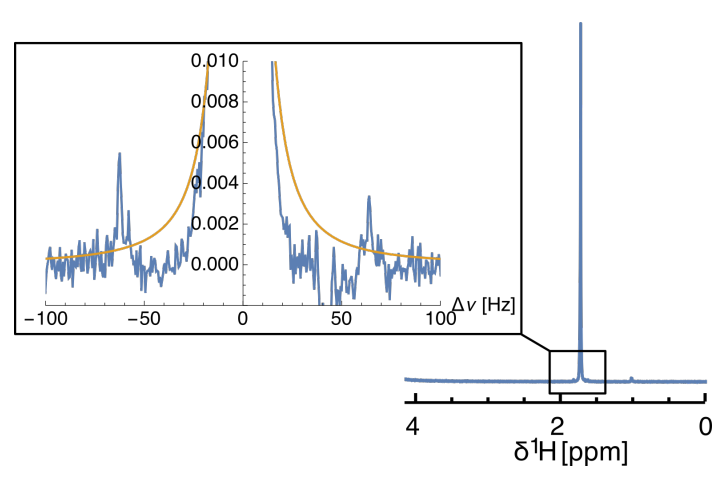

Figure 9: Spectrum of $130 \mathrm{mM}$ sodium acetate dissolved in water acquired in 32 scans with water presaturation. Carbon satellites can be seen in the expanded base of the acetate line. The orange curve shows a lorentzian lineshape with full width at half maximum of 3.35 $\mathrm{Hz}$.

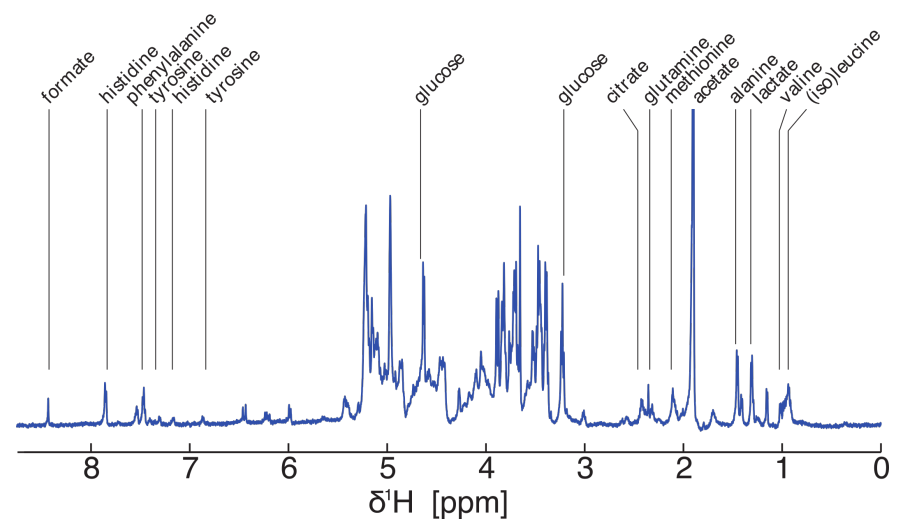

Figure 10: Spectrum of culture growth media recorded in 256 scans with water presaturation. The total experimental time was 20 min for $2.5 \mu \mathrm{l}$ sample volume.
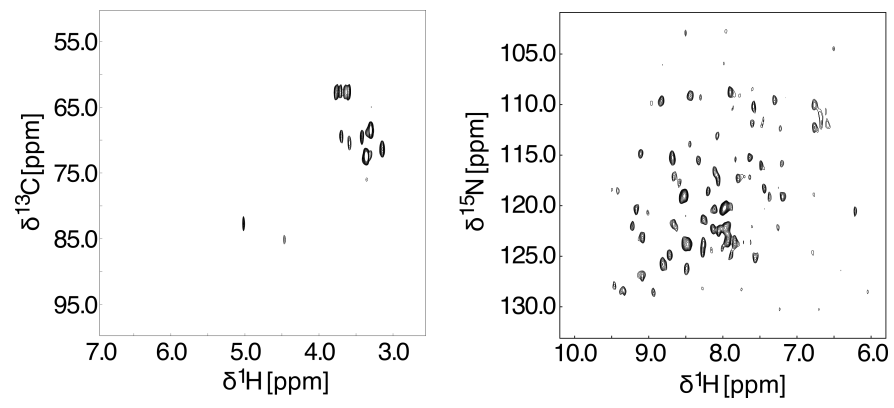

Figure 11: ${ }^{1} \mathrm{H}_{-}{ }^{13} \mathrm{C}$ HSQC spectrum of ${ }^{13} \mathrm{C}$ labeled glucose (left) and ${ }^{1} \mathrm{H}-{ }^{15} \mathrm{~N}$ HSQC of ${ }^{15} \mathrm{~N}$ labeled Ubiquitin (right) both acquired from $2.5 \mu \mathrm{l}$ sample volume at $14.1 \mathrm{~T}$. Glucose HSQC spectrum was acquired in 12 minutes from a $100 \mathrm{mM}$ sample. Ubiquitin HSQC spectrum was acquired in 400 minutes from a $1 \mathrm{mM}$ sample.

lineshape with full width at half maximum of $3.35 \mathrm{~Hz}$, corresponding to $0.0056 \mathrm{ppm}$. This is very similar to the resolution reported by Finch et al. at $300 \mathrm{MHz}$. While the residual cause of broadening is still under investigation, we have reason to suspect imperfections in the fabrications of the PMMA microfluidic devices to play a major role. Efforts to mitigate these and to achieve more highly resolved proton spectra are currently underway in our laboratory and will be reported at a later occasion. The limit of detection $n L O D_{\omega}=\frac{3 n \sqrt{\Delta t}}{S N R}$ ( $n$ is the number of spins and $\Delta t$ is $1 /$ linewidth of the observed resonance) is $1.4 \mathrm{nmol}$ $\mathrm{s}^{1 / 2}$ for protons at $600 \mathrm{MHz}$, slightly better than the value reported for a smaller detector by Finch et al. [32].

Even though the resolution of the proton spectra still needs to be improved, it is sufficient to analyse complex mixtures. The proton spectrum of DMEM (Dulbecco modified Eagle's medium) shown in Fig. 10 demonstrates the suitability of this probe for metabolomic studies of microfluidic cultures of cells, tissues, and small organisms. Fig. 11 demonstrates proton-detected double resonance experiments in the current setup. The left panel shows a ${ }^{13} \mathrm{C}-{ }^{1} \mathrm{H}$ heteronuclear single quantum correlation (HSQC) spectrum of ${ }^{13} \mathrm{C}$-glucose and the right panel shows a ${ }^{15} \mathrm{~N}$ ${ }^{1} \mathrm{H}$-HSQC spectrum of ${ }^{15} \mathrm{~N}$-ubiquitin $(17 \mu \mathrm{g})$. All expected cross peaks are present in the spectrum. The ability to obtain meaningful ${ }^{15} \mathrm{~N}-{ }^{1} \mathrm{H}$ correlation spectra from such small samples could prove very useful in the context of proteins that are difficult to express and therefore only available in small quantities. The integration of protein NMR and microfluidic technology also opens new possibilities for high-throughput kinetics and binding studies.

The probe can also be used to acquire micro magnetic resonance (MR) images using a Bruker micro-gradient unit. To this effect, the aluminium probe sleeve must be replaced by an electrically insulating material in order to avoid image distortions due to eddy currents in the sleeve. A shorter aluminium tube with 3D printed top part (made from ABS plastic) to match the probe height was used. Fig. 12 shows MR images of mouse liver tissue slice immersed in DMEM 


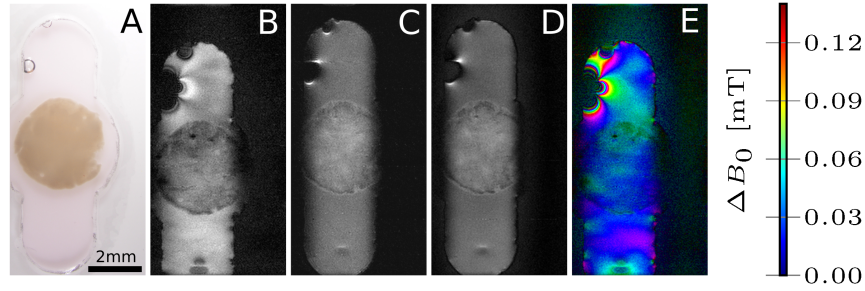

Figure 12: MR images of a freshly cut liver slice from a mouse. A optical micrograph; B: Gradient echo image acquired in 1 minute; C: RARE8 image acquired in 8 minutes; D: Spin echo image acquired in 30 minutes; E: magnetic field map.

cell growth medium. The slice was placed in a microfluidic device with circular sample chamber centred at the detector location, as shown in Fig. 12(A). The image shown in Fig. 12(B) was acquired using a FLASH pulse sequence with; the lateral dimensions of all MR images in Fig. 12 are $512 \times 512$ pixels at a resolution of $30 \mu \mathrm{m}$. Fig. $12(\mathrm{C})$ shows a RARE image using the same parameters. A spin echo image is shown in Fig. 12(D). Finally, Fig. 12(E) shows a $B_{0}$ field map, which has been obtained by acquiring two separate FLASH images.

\section{Conclusions}

We have presented a novel modular NMR probe assembly for generic high sensitivity microfluidic NMR experiments. The probe is built from the readily accessible materials, using only a minimum of custom-made parts. The modularity of the probe enables to use different detectors tailored for specific experimental requirements on the same probe skeleton. The detector itself is made from easily available PCB materials. The use of PCB technology offers great flexibility, and allows to modify the probe circuit and design without affecting the probe geometry. The probe has excellent $\mathrm{B}_{1}$ homogeneity and its sensitivity is in line with the best performances that have been reported in this range of probe volumes.

Using simple microfluidic devices made from PMMA sheets by laser cutting, spectral resolution around $0.005 \mathrm{ppm}$ is achieved in proton spectra at $600 \mathrm{MHz}$. This is sufficient for primary metabolic studies, but some applications could benefit from better resolution. The probe has already been used for various experiments including reaction monitoring [38], micro-imaging of mouse liver tissue slices, and metabolic studies of mammalian cells. The probe is designed for double resonance, and acquisition of protondetected heteronuclear correlation experiments has been demonstrated for small molecules as well as proteins. In the future we intend to add a field-frequency lock channel, as well as additional functionality for advanced microfluidic perfusion with temperature and flow control.

\section{Acknowledgement}

This research was supported by the "TISuMR" project, funded through "Future and Emerging Technologies" (FETOPEN) call of the EU Horizon 2020 research framework. The authors would like to thank Mr. William Hale for help with making microfluidic devices, Dr. Katrin Deinhardt for providing the mouse liver tissue, Dr. Bishnubrata Patra for tissue slicing and Dr. Jörn Werner for providing the labelled ubiquitin sample and for his support in the acquisition and processing of the HSQC spectrum. 


\section{References}

[1] Frank S Pullen, Alistair G Swanson, Michael J Newman, and Don S Richards. 'on-line' liquid chromatography/nuclear magnetic resonance mass spectrometry a powerful spectroscopic tool for the analysis of mixtures of pharmaceutical interest. Rapid communications in mass spectrometry, 9(11):1003-1006, 1995.

[2] Brian P Hills. Magnetic resonance in food science. In Dynamics of solutions and fluid mixtures by NMR. 1994.

[3] DL Rabenstein and DA Keire. Quantitative chemical analysis by nmr. In Modern NMR techniques and their application in chemistry. 1991.

[4] Kurt Wüthrich. Protein structure determination in solution by $\mathrm{nmr}$ spectroscopy. Journal of Biological Chemistry, 265(36):22059-22062, 1990.

[5] John L Markley, Rafael Brschweiler, Arthur S Edison, Hamid R Eghbalnia, Robert Powers, Daniel Raftery, and David S Wishart. The future of nmr-based metabolomics. Current Opinion in Biotechnology, 43:34 - 40, 2017. Analytical biotechnology.

[6] Teresa W.-M. Fan and Andrew N. Lane. Applications of nmr spectroscopy to systems biochemistry. Progress in Nuclear Magnetic Resonance Spectroscopy, 92-93:18 - 53, 2016.

[7] Michael Maiwald, Holger H Fischer, Young-Kyu Kim, Klaus Albert, and Hans Hasse. Quantitative high-resolution on-line nmr spectroscopy in reaction and process monitoring. Journal of Magnetic Resonance, 166(2):135-146, 2004.

[8] George M Whitesides. The origins and the future of microfluidics. Nature, 442(7101):368, 2006.

[9] Daniel Mark, Stefan Haeberle, Günter Roth, Felix Von Stetten, and Roland Zengerle. Microfluidic lab-on-a-chip platforms: requirements, characteristics and applications. In Microfluidics Based Microsystems, pages 305-376. Springer, 2010.

[10] Jamil El-Ali, Peter K. Sorger, and Klavs F. Jensen. Cells on chips. Nature, 442:403 EP -, 072006.

[11] Helene Andersson and Albert van den Berg. Microfluidic devices for cellomics: a review. Sensors and Actuators B: Chemical, 92(3):315 - 325, 2003.

[12] Hiroshi Kimura, Takatoki Yamamoto, Hitomi Sakai, Yasuyuki Sakai, and Teruo Fujii. An integrated microfluidic system for long-term perfusion culture and on-line monitoring of intestinal tissue models. Lab Chip, 8:741-746, 2008.

[13] He Wen, Yong Jin An, Wen Jun Xu, Keon Wook Kang, and Sunghyouk Park. Real-time monitoring of cancer cell metabolism and effects of an anticancer agent using $2 \mathrm{~d}$ in-cell nmr spectroscopy. Angewandte Chemie International Edition, 54(18):5374-5377, 2015.

[14] Dean L. Olson, Timothy L. Peck, Andrew G. Webb, Richard L. Magin, and Jonathan V. Sweedler. High-resolution microcoil 1h-nmr for mass-limited, nanoliter-volume samples. Science, 270(5244):1967-1970, 1995.

[15] Vlad Badilita, Robert Ch. Meier, Nils Spengler, Ulrike Wallrabe, Marcel Utz, and Jan G. Korvink. Microscale nuclear magnetic resonance: a tool for soft matter research. Soft Matter, 8:10583-10597, 2012.

[16] Sergey S. Zalesskiy, Ernesto Danieli, Bernhard Blmich, and Valentine P. Ananikov. Miniaturization of nmr systems: Desktop spectrometers, microcoil spectroscopy, and $\mathrm{nmr}$ on a chip for chemistry, biochemistry, and industry. Chemical Reviews, 114(11):5641-5694, 2014. PMID: 24779750.

[17] R. Subramanian, M.M. Lam, and A.G. Webb. Rf microcoil design for practical nmr of mass-limited samples. Journal of Magnetic Resonance, 133(1):227 - 231, 1998.

[18] Elad Harel, Christian Hilty, Katherine Koen, Erin E. McDonnell, and Alex Pines. Time-of-flight flow imaging of twocomponent flow inside a microfluidic chip. Phys. Rev. Lett., 98:017601, Jan 2007.

[19] Yael Maguire, Isaac L. Chuang, Shuguang Zhang, and Neil Gershenfeld. Ultra-small-sample molecular structure detection using microslot waveguide nuclear spin resonance. Proceedings of the National Academy of Sciences, 104(22):9198-9203, 2007.
[20] Hans Georg Krojanski, Jrg Lambert, Yilmaz Gerikalan, Dieter Suter, and Roland Hergenrder. Microslot nmr probe for metabolomics studies. Analytical Chemistry, 80(22):8668-8672, 2008.

[21] K. Ehrmann, M. Gersbach, P. Pascoal, F. Vincent, C. Massin, D. Stamou, P.-A. Besse, H. Vogel, and R.S. Popovic. Sample patterning on nmr surface microcoils. Journal of Magnetic Resonance, 178(1):96 - 105, 2006.

[22] P.J.M. van Bentum, J.W.G. Janssen, A.P.M. Kentgens, J. Bart, and J.G.E. Gardeniers. Stripline probes for nuclear magnetic resonance. Journal of Magnetic Resonance, 189(1):104 - 113, 2007.

[23] SGJ van Meerten, P Jan M van Bentum, and Arno PM Kentgens. Shim-on-chip design for microfluidic nmr detectors. Analytical chemistry, 90(17):10134-10138, 2018.

[24] E. Montinaro, M. Grisi, M. C. Letizia, L. Peth, M. A. M. Gijs, R. Guidetti, J. Michler, J. Brugger, and G. Boero. 3d printed microchannels for sub-nl nmr spectroscopy. PLOS ONE, 13(5):1-17, 052018.

[25] Ying Chen, Hardeep S Mehta, Mark C Butler, Eric D Walter, Patrick N Reardon, Ryan S Renslow, Karl T Mueller, and Nancy M Washton. High-resolution microstrip nmr detectors for subnanoliter samples. Physical Chemistry Chemical Physics, 19(41):28163-28174, 2017.

[26] Anna Jo Oosthoek-de Vries, Jacob Bart, Roald M Tiggelaar, Johannes WG Janssen, P Jan M van Bentum, Han JGE Gardeniers, and Arno PM Kentgens. Continuous flow $1 \mathrm{~h}$ and $13 \mathrm{c}$ $\mathrm{nmr}$ spectroscopy in microfluidic stripline nmr chips. Analytical chemistry, 89(4):2296-2303, 2017.

[27] Jacob Bart, Ard J Kolkman, Anna Jo Oosthoek-de Vries, Kaspar Koch, Pieter J Nieuwland, Hans Janssen, Jan van Bentum, Kirsten AM Ampt, Floris PJT Rutjes, Sybren S Wijmenga, et al. A microfluidic high-resolution nmr flow probe. Journal of the American Chemical Society, 131(14):5014-5015, 2009.

[28] J Bart, JWG Janssen, PJM Van Bentum, APM Kentgens, and Johannes GE Gardeniers. Optimization of stripline-based microfluidic chips for high-resolution nmr. Journal of magnetic resonance, 201(2):175-185, 2009.

[29] C Massin, F Vincent, A Homsy, K Ehrmann, G Boero, P-A Besse, A Daridon, E Verpoorte, NF De Rooij, and RS Popovic. Planar microcoil-based microfluidic nmr probes. Journal of Magnetic Resonance, 164(2):242-255, 2003.

[30] N Spengler, A Moazenzadeh, R Ch Meier, V Badilita, J G Korvink, and $\mathrm{U}$ Wallrabe. Micro-fabricated helmholtz coil featuring disposable microfluidic sample inserts for applications in nuclear magnetic resonance. Journal of Micromechanics and Microengineering, 24(3):034004, 2014.

[31] Nils Spengler, Jens Hfflin, Ali Moazenzadeh, Dario Mager, Neil MacKinnon, Vlad Badilita, Ulrike Wallrabe, and Jan G. Korvink. Heteronuclear micro-helmholtz coil facilitates m-range spatial and sub-hz spectral resolution nmr of nl-volume samples on customisable microfluidic chips. PLOS ONE, 11(1):1-16, 01 2016.

[32] Graeme Finch, Ali Yilmaz, and Marcel Utz. An optimised detector for in-situ high-resolution $\mathrm{nmr}$ in microfluidic devices. Journal of Magnetic Resonance, 262:73 - 80, 2016.

[33] Ian Swyer, Sebastian von der Ecken, Bing Wu, Amy Jenne, Ronald Soong, Franck Vincent, Daniel Schmidig, Thomas Frei, Falko Busse, Henry J Stronks, et al. Digital microfluidics and nuclear magnetic resonance spectroscopy for in situ diffusion measurements and reaction monitoring. Lab on a Chip, 2019.

[34] Ali Yilmaz and Marcel Utz. Characterisation of oxygen permeation into a microfluidic device for cell culture by in situ $\mathrm{nmr}$ spectroscopy. Lab Chip, 16:2079-2085, 2016.

[35] Matthias C. Wapler, Jochen Leupold, Iulius Dragonu, Dominik von Elverfeld, Maxim Zaitsev, and Ulrike Wallrabe. Magnetic properties of materials for $\mathrm{mr}$ engineering, micro-mr and beyond. Journal of Magnetic Resonance, 242:233 - 242, 2014.

[36] John F. Schenck. The role of magnetic susceptibility in magnetic resonance imaging: Mri magnetic compatibility of the first and second kinds. Medical Physics, 23(6):815-850, 1996. 
[37] Richard Finch Graeme. Optimised detectors for integration of NMR with microfluidic devices. PhD thesis, University of Southampton, February 2017.

[38] Hongxun Fang, Yibin Sun, Xinchang Wang, Manvendra Sharma, Zhong Chen, Xiaoyu Cao, Marcel Utz, and Zhongqun Tian. Probing the kinetics in supramolecular chemistry and molecular assembly by microfluidic-nmr spectroscopy. Science China Chemistry, 61(11):1460-1464, Nov 2018. 